\title{
ON QUASI I-OPENNESS AND QUASI I-CONTINUITY
}

\author{
M. E. ABD EL-MONSEF*, R. A. MAHMOUD** AND A. A. NASEF***
}

\begin{abstract}
A space $(X, \tau, I)$ consisting of a nonempty set $X$ with a topology $\tau$ and an ideal $I$ of subsets of $X$ which has heredity and finite additivity properties. In this paper the quasi $I$-open and quasi $I$-closed sets are presented. Utilizing these new concepts the class of quasi $I$-continuous functions have been obtained. Both of quasi $I$-openness and quasi $I$-continuity is considered as a generalization of those $I$-openness and $I$-continuity. However, numerous topological properties of these new notions have been discussed as well as many of their known results have been improved.
\end{abstract}

\section{Introduction}

In the beginning of ninetieth, Jankovic and Hamlett [5] have define the concept of $I$-open set via the local function which was given by Vaidanathaswamy [15] in 1945. The latter concept was also established utilizing the concept of an ideal whose topic in general topological spaces was treated in the classic text by Kuratwski [6] in 1933. Recently, in 1992, Abd El-Monsef et al., [2] studied a number of $I$-openness properties not only but also they obtained the both of $I$-closed sets and $I$-continuous functions and investigated several of their properties. Therefore, we devoted this paper to generalize the $I$-openness by presenting the class of quasi $I$-open sets. The class of a: quasi $I$-continuous function have been also established and studied. The connections between these new concepts with the corresponding types are discussed. Some of their characterizations and other numerous properties are studied. The importance of these new notions is shown as generalizations of their corresponding known types, this helps us to strengthen some results in $[2,5]$.

\section{Preliminaries}

Throughout the present paper, $(X, \tau)$ and $(Y, \sigma)$ are denoted by the topological spaces or simply spaces on which no separation properties are assumed unless the needed ones its will be explicitly stated. In $(X, \tau)$, the usual closure and the interior of any $W \subseteq X$ will be denoted by $\tau$-cl $(W)$ and $\tau$-int $(W)$, respectively. The notion $(X, \tau, I)$ means a space $(X, \tau)$ with an ideal $I$ on $X$ which is a nonempty subclass of a power set $P(X)$ having the

Received April 20, 1998; revised January 26, 1999.

2000 Mathematics Subject Classification. 54A10, 54C10.

Key words and phrases. Ideal, quasi $I$-open set, quasi $I$-continuous function. 
heredity and finite additivity properties i.e. $I \subseteq P(X)$ is an ideal on $X$ if $W \in I$ and $S \subseteq$ $W$, then $S \in I$ and also if $W \in I$ and $S \in I$, then $W \cup S \in I$ [6]. Sometimes an ideal called a dual filter [4]. For any $W \subseteq X$ in $(X, \tau, I), W^{*}(I, \tau)$ or simply $W^{*}(I)$ means the local function of $W$ with respect to $I$ and $\tau$ and is defined by $W^{*}(I, \tau)=\{x \in X: W \cup N \notin I$, $N \in \tau(x)\}$ [15] where $\tau(x)$ denotes the open-neighbourhood of $x$. The simplest ideals are $\{\phi\}$ and $P(X)$ which satisfied $\{\phi\} \subseteq I \subseteq P(X)$, for any $I$ on $X$. Also, between the useful ideals is the collection of all nowhere dense subsets of $(X, \tau)$ which is an ideal and denoted by $I_{n}$. i.e. $I_{n}=\{W \subseteq X: \tau-\operatorname{int}(\tau-\operatorname{cl}(W)=\phi\}$. For $(X, \tau, I)$, its say that the topology $\tau$ is compatible with an ideal $I$, denoted $\tau \sim I$, if the following holds: for every $W \subseteq X$ and every $x \in W$ there exists $N \in \tau(x)$ such that $N \cap W \in I$, then $W \in I$ [13]. $W \subseteq X$ is said to be $I$-open [5] (resp. semi-open [8], $\alpha$-set [12], preopen [10], $\beta$-open [1]) if $W \subseteq \tau$-int $(W)^{*}$ (resp. $W \subseteq \tau-\operatorname{cl}(\tau-\operatorname{int}(W)), W \subseteq \tau-\operatorname{int}(\tau-\operatorname{cl}(\tau-\operatorname{int}(W)))$, $W \subseteq \tau-\operatorname{int}(\tau-\operatorname{cl}(W)), W \subseteq \tau-\operatorname{cl}(\tau-\operatorname{int}(\tau-\operatorname{cl}(W))))$ while their complements are the corresponding types of closeness. But the classes of all previous types of openness in $(X, \tau)$ are denoted by $I O(X, \tau), S O(X, \tau), \tau^{\alpha}, P O(X, \tau)$ and $\beta O(X, \tau)$, respectively. a function $f:(X, \tau) \rightarrow(Y, \sigma)$ is called $I$-continuous [2], semicontinuous [8], precontinuous [10] and $\beta$-continuous [1] if the inverse image of each open set is $I$-open, semiopen, preopen and $\beta$-op3n, respectively. While, $f:(X, \tau) \rightarrow(Y, \sigma)$ is $\beta$-irresolute [9], if the inverse image of each $\beta$-op3n set in $(Y, \sigma)$ is also $\beta$-open in $(X, \tau)$. A space $(X, \tau)$ is said to be $I$-compact $[11,14]$ if for every open cover $\left\{U_{1}: i \in \nabla\right\}$ of $X$, there exists a finite $\nabla_{0}$ of $\nabla$ such that $X-\cup\left\{U_{i}: i \in \nabla_{0}\right\} \in I$.

\section{Quasi I-open Sets}

Definition 1. In $(X, \tau, I), W \subseteq X$ is said to be quasi $I$-open if $W \subseteq \tau-\operatorname{cl}(\tau-$ $\left.\operatorname{int}\left(W^{*}\right)\right),(X-W)$ is called quasi $I$-closed.

When there is no chance of confusion, the collection of all quasi $I$-open sets of $(X, \tau)$ will be denoted by $Q I O(X, \tau)$. Also, $Q I O(X, x)$ means the class of all quasi $I$-open sets containing $x \in X$.

The connections between quasi $I$-openness with some other corresponding types have been given throughout the following implication.

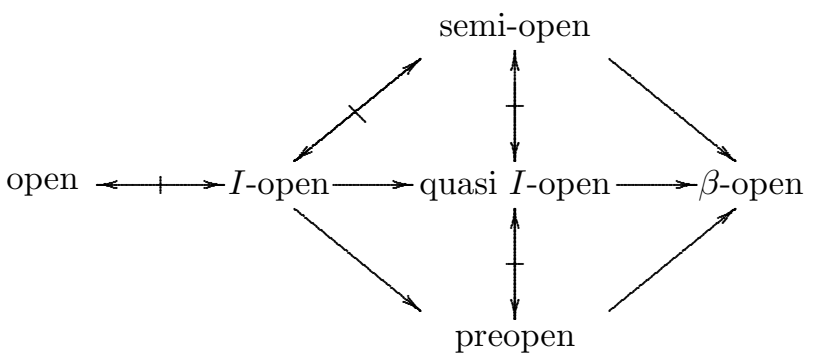


The above relationships can not be reversable as the next examples illustrate, while others were given in $[1,2]$.

Example 1. Let $X=\{a, b, c, d\}$ with a topology $\tau=\{X, \phi,\{c\},\{a, b\},\{a, b, c\}\}$ and the two ideals $I=\{\phi,\{c\},\{d\},\{c, d\}\}$ and $J=\{\phi,\{a\}\}$, then we can show the followings;

(i) $W=\{a, d\} \in Q I O(X, \tau)$ but it is neither $I$-open nor preopen.

(ii) While $S=\{a, c\}$ is precopen but is is not quasi $I$-open.

(iii) The set $\{c, d\} \in S O(X, \tau)$ but not $I$-open.

(iv) Each of $\{b\},\{b, c, d\}$ is $J$-open and therefore quasi $J$-open but not semi-open.

Example 2. If $X=\{a, b, c\}$ and $\tau=\{X, \phi,\{a\},\{c\},\{a, c\}\}$ with $I=\{\phi,\{b\},\{c\}$, $\{b, c\}\}$ and $J$ as in Example 1. Then we have;

(i) Both of $\{c\}$ and $\{a, c\}$ are semi-open but not quasi $I$-open.

(ii) The set $W=\{a, b\} \in \beta O(X, \tau)$ but $W \notin Q J O(X, \tau)$.

Remark 1. One can shows that the closeness cases follow by the complement operation of the openness types in the above examples.

Proposition 1. Arbitrary union of quasi I-open sets is also quasi I-open.

Proof. Let $(X, \tau, I)$ be any space and $W_{i} \in Q I O(X, \tau)$ for $i \in \nabla$, this means that for each $i \in \nabla, W_{i} \subseteq \tau-\operatorname{cl}-\left(\tau-\operatorname{int}\left(W_{i}^{*}\right)\right)$ and $s o \cup_{i \in \nabla} \tau-\operatorname{cl}\left(\tau-\operatorname{int}\left(W_{i}^{*}\right)\right)=$ $\tau-\operatorname{cl}\left(\tau-\operatorname{int}\left(W_{i}^{*}\right)\right) \subseteq \tau-\operatorname{cl}\left(\tau-\operatorname{int}\left(\cup_{i \in \nabla} W\right)^{*}\right)$. Hence $\cup_{i \in \nabla} W_{i} \in Q I O(X, \tau)$.

Lemma 1. [4] Let a space $(X, \tau)$ with an ideal $I_{n}$ on $X$. Then $X$ coincides with its local function (i.e. $X=X^{*}$ ).

Above two results a useful to obtain the following theorem which is strengthened for the corresponding one in $[2,5]$.

Theorem 1. For a space $\left(X, \tau, I_{n}\right)$, the class $Q I O(X, \tau)$ forms a supratopology.

Proof. Follows by the fact $\phi^{*}=\left\{x \in X: \phi \cap N_{x} \notin i_{n}\right\}=\phi$ and both of Lemma 1 and Proposition 1.

The above theorem leads us to present the following question: Is $Q I O(X, \tau)$ form a topology? The answer is no for the next remark.

Remark 2. A finite intersection of quasi $I$-open sets need not in general quasi $I$-open, as Example 3 shows.

Example 3. Let $X=\{a, b, c, d\}$ and $\tau=\{X, \phi,\{a, b\},\{a, b, c\}\}$ with $I=\{\phi,\{c\},\{d\}$, $\{c, d\}\}$. We deduce that the two sets $W_{1}=\{a, c\}$ and $W_{2}=\{b, c\}$ are quasi $I$-open while their intersection does not.

The above remark, turns our attention to establish the following result. 
Proposition 2. In $(X, \tau, I)$, if $U \in \tau$ and $W \in Q I O(X, \tau)$, then $U \cap W$ is quasi I-open.

Proof. By hypothesis and the fact that $U \cap \tau-\operatorname{cl}(W) \subseteq \tau-\operatorname{cl}(U \cap W)$, we have, $U \cap W \subseteq U \cap \tau-\operatorname{cl}\left(\tau-\operatorname{int}\left(W^{*}\right)\right) \subseteq \tau-\operatorname{cl}\left(U \cap \tau-\operatorname{int}\left(W^{*}\right)\right)$. Then by both of Theorem (4.1) in [5] and Theorem (2.6) in [2] we get $U \cap W \subseteq \tau-\operatorname{cl}\left(\tau-\operatorname{int}(U \cap W)^{*}\right)$. Hence the result.

If the openness of $U$ in the above result have generalized by an $\alpha$-set, then we obtain.

Theorem 2. The intersection of quasi I-open with an $\alpha$-set is $\beta$-open.

Proof. Let $(X, \tau, I)$ be a space, $W \in Q I O(X, \tau)$ and $G \in \tau^{\alpha}$. Then $W \cap G \subseteq \tau-$ $\operatorname{cl}\left(\tau-\operatorname{int}\left(W^{*}\right)\right) \cap \tau-\operatorname{int}(\tau-\operatorname{cl}(\tau-\operatorname{int}(G))) \subseteq \tau-\operatorname{cl}\left(\tau-\operatorname{int}\left(W^{*}\right) \cap \tau-\operatorname{int}(\tau-\operatorname{cl}(\tau-\operatorname{int}(G)))=\right.$ $\tau-\operatorname{cl}\left(\tau-\operatorname{int}\left(\tau-\operatorname{int}\left(W^{*}\right) \cap \tau-\operatorname{cl}(\tau-\operatorname{int}(G))\right) \subseteq \tau-\operatorname{cl}\left(\tau-\operatorname{int}\left(\tau-\operatorname{cl}\left(W^{*} \cap \tau-\operatorname{int}(G)\right)\right)\right.\right.$ $[4$, theorem $(2.3)] \subseteq \tau-\operatorname{cl}(\tau-\operatorname{int}(\tau-\operatorname{cl}(W) \cap \tau-\operatorname{int}(G)))) \subseteq \tau-\operatorname{cl}(\tau-\operatorname{int}(\tau-\operatorname{cl}(\tau-$ $\operatorname{cl}(W \cap G))))=\tau-\operatorname{cl}(\tau-\operatorname{int}(\tau-\operatorname{cl}(W \cap(G))))$, which completes the proof.

Recall that in $(X, \tau, I)$ any $W \subseteq X$ is $*$-dense-in-itself [3], $*$-closed [15] and $*$-perfect [3] if $W \subseteq W^{*}, W^{*} \subseteq W$ and $W=W^{*}$, respectively.

Proposition 3. The following statements are verified

(i) For $(X, \tau,\{\phi\}), Q I O(X, \tau)=\beta O(X, \tau)$.

(ii) For $(X, \tau, P(X)), Q I O(X, \tau)=I O(X, \tau)$.

(iii) For any $(X, \tau, I)$ each quasi I-open (resp. semi-open) which it is *-closed (resp. *-dense-in-itself) is semi-open (resp. quasi I-open).

Since $*$-dense-in-itself and $*$-closeness together of any $W \subseteq X$ in $(X, \tau, I)$ equivalent with the $*$-perfect property of $W$ in the same space. Then the two classes $Q I O(X, \tau)$ and $S O(X, \tau)$ are coincides with each other, if each member of term is *-perfect or both of $*$-dense-in-itself and $*$-closed. In other words, the two parts of statement (iii) previously equivalent with: $Q I O(X, \tau)=S O(X, \tau)$ if $W \subseteq X$ is $*$-perfect for any $(X, \tau, I)$.

Lemma 2. [4] For each $W \subseteq X$ in $\left(X, \tau, I_{n}\right), W^{*}\left(I_{n}\right) \subseteq W$.

Proposition 4. In $\left(X, \tau, I_{n}\right)$, if $W \in Q I O(X, \tau)$, then it is semi-open.

Hence we can deduce that a quasi $I$-open set which is $*$-closed for any $(X, \tau, I)$ is equivalent with the quasi $I$-openness in $\left(X, \tau, I_{n}\right)$ which is useful to obtain the following.

Proposition 5. $(X, \tau, I)$ any preclosed set $W \subseteq X$ is regular-closed if one of the following hold:

(i) $W$ is $*$-closed and quasi $I$-open.

(ii) $W \in Q I O\left(X, \tau, I_{n}\right)$.

Proposition 6. In $(X, \tau, I)$, if $U \in \tau$ and $W \in Q I O(X, \tau)$. Then $U \cap W \in$ $Q I O(U, \tau \mid U)$. 
Proof. Since $U \cap W \subseteq U \cap \tau-\operatorname{cl}\left(\tau-\operatorname{int}\left(W^{*}\right)\right) \subseteq \tau-\operatorname{cl}\left(U \cap \tau-\operatorname{int}\left(W^{*}\right)\right) \cap$ $U=\operatorname{cl}_{U}\left(U \cap \tau-\operatorname{int}\left(W^{*}\right)\right)$. While $U \cap \tau-\operatorname{int}\left(W^{*}\right) \subseteq U$ which is open in $U$. i.e. $\operatorname{int}_{U}\left(U \cap \tau-\operatorname{int}(W)^{*}\right)=U \cap \tau-\operatorname{int}\left(W^{*}\right)$. Thus $U \cap W \subseteq \operatorname{cl}_{U}\left(\operatorname{int}_{U}\left(U \cap \tau-\operatorname{int}(W)^{*}\right)\right) \subseteq$ $\operatorname{cl}_{U}\left(\operatorname{int}_{U}\left(U \cap W^{*}\right)\right) \subseteq \operatorname{cl}_{U}\left(\operatorname{int}_{U}(U \cap W)^{*}\right)[4$, Theorem (2.3)]. Hence the result.

Proposition 7. For $(X, \tau, I)$ if $W \subseteq U \subseteq X, U \in \tau$ and $W \in Q I O(U, \tau \mid U)$. Then $W \in Q I O(X, \tau)$.

Proof. Since $W \subseteq \operatorname{cl}_{U}\left(\operatorname{int}_{U}\left(W^{*}\right)\right)$ and since $\operatorname{int}_{U}\left(W^{*}\right)$ is an open set in $U$, there exists $V \in \tau$ such that $\operatorname{cl}_{U}\left(W^{*}\right)=U \cap V \in \tau$. Hence $W \subseteq \operatorname{cl}_{U}(U \cap V)=\tau-\operatorname{cl}((U \cap V) \cap U)=$ $\tau-\operatorname{cl}(U \cap V)=\tau-\operatorname{cl}\left(\operatorname{int}_{U}\left(W^{*}\right)\right)=\subseteq \tau-\operatorname{cl}-\left(\tau-\operatorname{int}\left(W^{*}\right)\right)$. Thus $W \in Q I O(X, \tau)$.

Janković and Hamlett [5] have denoted by $I * J$ the extension of and ideal $I$ via other one $J$ which are given on the same nonempty set $X$ with respect to $(X, \tau)$ and defined as : $I * J=\left\{A \subseteq X: A^{*}(I) \in J\right\}$. They showed also that $I * J$ is an ideal on $X$ and $I \subseteq I * J$.

Lemma 3. [5] Let $(X, \tau, I)$ be a space with $I_{n} \subseteq I$ and $\tau \sim I$. Then for any other ideal $J$ on $X, \tau \sim(J * I)$.

For any $(X, \tau, I)$, the authors in [5] denote $\left(I * I_{n}\right)$ by $I^{\sim}$ which is a compatible extension of $I$ and it is also defined as $I^{\sim}=\left\{A \subseteq X: \tau-\operatorname{int}\left(A^{*}\right)=\phi\right\}$. This leads us to establish the following equivalent.

Theorem 3. Given $(X, \tau, I)$, any $W \subseteq X$ is quasi $I$-open iff $W \subseteq W^{*}\left(I^{\sim}\right)$.

Proof. Necessity, let $x \notin W^{*}\left(I^{\sim}\right)$, then there exists an open neighbourhood $N$ of $x$ such that $\tau-\operatorname{int}(N \cap W)^{*}(I)=\phi$. Since $N \cap W^{*}(I) \subseteq(N \cap W)^{*}$ (I) [7], then $N \cap \tau-\operatorname{int}\left(W^{*}(I)\right) \subseteq \tau-\operatorname{int}(N \cap W)^{*}(I)=\phi$. This means, $x \notin \tau-\operatorname{cl}\left(\tau-\operatorname{int}\left(W^{*}\right)\right)$ and by quasi $I$-openness of $W$ we get, $x \notin W$. Sufficiency, since $I_{n} \subseteq I^{\sim}$, then for any $W \subseteq X$, $W^{*}\left(I^{\sim}\right) \subseteq W^{*}\left(I_{n}\right)=\tau-\operatorname{cl}(\tau-\operatorname{int}(\tau-\operatorname{cl}(W)))$ [16]. If we put $W^{*}$ instead of $W$, the last inclusion gives $\left.\left(W^{*}\left(I^{\sim}\right)\right)^{*}\left(I^{\sim}\right) \subseteq \tau-\operatorname{cl}\left(\tau-\operatorname{int}\left(\tau-\operatorname{cl} W^{*}\left(I^{\sim}\right)\right)\right)\right)=\tau-\operatorname{cl}\left(\tau-\operatorname{int}\left(W^{*}\left(I^{\sim}\right)\right)\right)$, for $W^{*}\left(I^{\sim}\right)$ is closed [4, Theorem (2.3)]. By $I^{\sim} \sim \tau$, we get $\left(W^{*}\left(I^{\sim}\right)\right)=\left(W^{*}\left(I^{\sim}\right)\right)^{*}\left(I^{\sim}\right)$ [8]. Therefore, $W^{*}\left(I^{\sim}\right) \subseteq \tau-\operatorname{cl}\left(\tau-\operatorname{int}\left(W^{*}\left(I^{\sim}\right)\right)\right) \subseteq W^{*}\left(I^{\sim}\right)$. Hence $W \subseteq W^{*}\left(I^{\sim}\right) \subseteq$ $\tau-\operatorname{cl}\left(\tau-\operatorname{int}\left(W^{*}\left(I^{\sim}\right)\right)\right) \subseteq \tau-\operatorname{cl}\left(\tau-\operatorname{int}\left(W^{*}(I)\right)\right)$, for $I \subseteq I^{\sim}$. Therefore $W \in Q I O(X, \tau)$ and the proof is complete.

\section{Quasi $I$-continuous Functions}

Definition 2. A function $f:(X, \tau, I) \rightarrow(Y, \sigma)$ is called quasi $I$-continuous if for every $V \in \sigma, f^{-1}(V) \in Q I O(X, \tau)$.

The relationships between this new class of functions and some types of non-continuous ones are obtained as follows. 


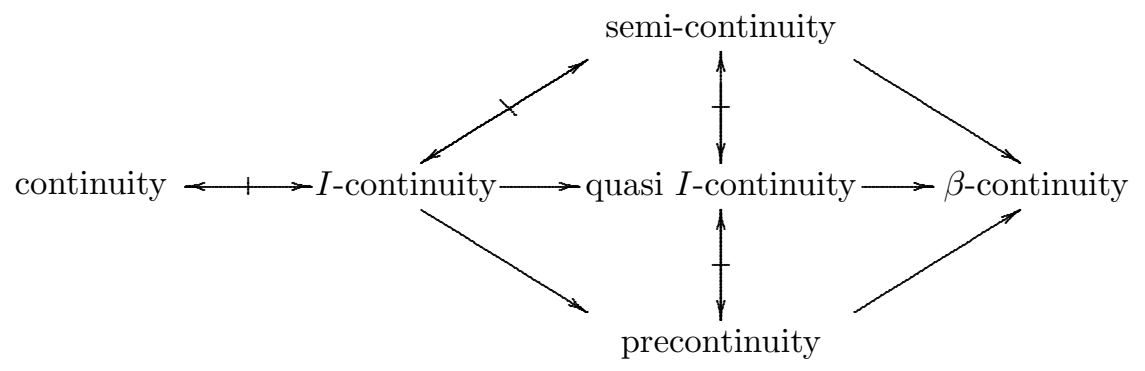

The converses need not be true in general as shown in Examples (2.1), (2.2) of [1], Examples (3.1), (3.2) and (3.3) of [2] and the following examples.

Example 4. Let $(X, \tau, J)$ be as in Example $1, Y=\{x, y, z\}$ and $\sigma=\{Y, \phi,\{y\},\{z\}$, $\{y, z\}\}$. A function $f:(X, \tau, J) \rightarrow(Y, \sigma)$ which is defined by $f(a)=f(b) x, f(c)=$ $f(d)=z$ is quasi $I$-continuous but not $I$-continuous.

Example 5. For a Serpniski space $(X, \tau)$ with an ideal $I=\{\phi,\{0\}\}$ and $Y=\{a, b, c\}$ with a topology $\sigma=\{Y, \phi,\{b\},\{a, b\}\}$. A function $f:(X, \tau, I) \rightarrow(Y, \sigma)$ which is defined by $f(0)=b, f(1)=a, f$ is $\beta$-continuous but not quasi $I$-continuous.

Example 6. Let $X, \tau$ be as in Example 3 with $I=\{\phi,\{c\},\{d\},\{c, d\}\}$. And $\sigma=$ $\{X, \phi,\{a\},\{a, b\},\{a, b, c\}\}$. A function $f:(X, \tau, I) \rightarrow(X, \sigma)$ which is defined by $f(a)=a$ and $f(b)=f(c)=f(d)=c$, is quasi $I$-continuous and $I$-continuous but not semicontinuous.

Example 7. Let $X=\{a, b, c\}, \tau=\{X, \phi,\{a\},\{b, c\}\}, \sigma=\{X, \phi,\{a\},\{a, b\}\}$, and $I=\{\phi,\{a\}\}$. Define $f:(X, \tau, I) \rightarrow(X, \sigma)$ as follows $f(a)=a$ and $f(b)=f(c)=b$. Then $f$ is semi-continuous but it is neither quasi $I$-continuous nor $I$-continuous.

Example 8. Let $X=\{a, b, c, d, e\}, \tau=\{X, \phi,\{a\},\{b\},\{a, b\},\{b, c\},\{a, b, c\}\}$ with $I=\{\phi,\{b\},\{c\},\{d\},\{b, c\},\{b, d\},\{c, d\},\{b, c, d\}\}$ and $Y=\{x, y, z\}, \sigma=\{Y, \phi,\{x\}\}$. One can shows $f:(X, \tau, I) \rightarrow(Y, \sigma)$ define by $f(a)=f(b)=x, f(c)=y$ and $f(d)=$ $f(e)=z$, is precontinuous but not quasi $I$-continuous.

Example 9. Let $X=\{a, b, c, d\}, \tau=\{X, \phi,\{c\},\{a, b\},\{a, b, c\}\}, I=\{\phi,\{a\}\}, Y=$ $\{p, q, r\}, \sigma=\{Y, \phi,\{q\},\{r\},\{q, r\}\}$ and $f:(X, \tau, I) \rightarrow(Y, \sigma)$ such that $f(a)=f(b)=p$ and $f(c)=f(d)=r$. Then $f$ is quasi $I$-continuous but not precontinuous.

Proposition 8. The following statements are true.

(i) $f:\left(X, \tau_{A}^{*}(I), I\right) \rightarrow(Y, \sigma)$ is quasi $I$-continuous if it is continuous, where $\tau_{A}^{*}(I)$ is the topology generated by $Q I O(X, \tau)$.

(ii) An quasi I-continuous function $f:\left(X, \tau, I_{n}\right) \rightarrow(Y, \sigma)$ is semi-continuous.

(iii) If $f:(X, \tau, I) \rightarrow(Y, \sigma)$ is quasi I-continuous (resp. semi-continuous) and for each $V \in \sigma, f^{-1}(V)$ is ${ }^{*}$-closed (resp. *-dense-in-itself) then $f$ is semi-continuous (resp. quasi I-continuous). 
Proposition 9. The following equivalents are verify.

(i) $f:(X, \tau,\{\phi\}) \rightarrow(Y, \sigma)$ is quasi I-continuous iff it is $\beta$-continuous.

(ii) $f:(X, \tau, P(X)) \rightarrow(Y, \sigma)$ is quasi I-continuous iff it is I-continuous.

(iii) Quasi I-continuity of a function $f:(X, \tau, I) \rightarrow(Y, \sigma)$ coincides with semi-continuity if for each $V \in \sigma, f^{-1}(V)$ is *-perfect or $f^{-1}(V)$ is both *-dense-in-itself and *closed.

Some equivalents of quasi $I$-continuity deduce throughout the next obviously result.

Theorem 4. For a function $f:(X, \tau, I) \rightarrow(Y, \sigma)$, the following are equivalent:

(i) $f$ is quasi I-continuous.

(ii) The inverse image of each closed set in $(Y, \sigma)$ is quasi I-closed.

(iii) For each $x \in X$ and each $V \in \sigma$ containing $f(x)$, there exists $W \in Q I O(X, \tau)$ containing $x$ such that $f(W) \subseteq V$.

Proposition 10. For the functions $f:(X, \tau, I) \rightarrow(Y, \sigma, J)$ and $g:(Y, \sigma, J) \rightarrow(Z, \mu)$, the followings are hold:

(i) If $f$ is quasi I-continuous and $g$ is continuous, then (gof) is quasi I-continuous.

(ii) (gof) is $\beta$-continuous, if $f$ is $\beta$-irresolute and $g$ is quasi I-continuous.

Theorem 5. The restriction function of almost I-continuity by an open set is also.

Proof. Let $f:(X, \tau, I) \rightarrow(Y, \sigma)$ be a quasi $I$-continuous function and $U \in \tau$. To show that $f \mid U$ is quasi $I$-continuous, let $V \in \sigma$, then $f^{-1}(V) \in Q I O(X, \tau)$. Proposition 6 , gives $(f \mid U)^{-1}(V)=U \cap f^{-1}(V) \subseteq U \cap \tau-\operatorname{cl}\left(\tau-\operatorname{int}\left(f^{-1}(V)\right)^{*}\right) \subseteq \tau-\operatorname{cl}[U \cap \tau-$ $\left.\operatorname{int}\left(f^{-1}(V)\right)^{*}\right] \subseteq \tau-\operatorname{cl}\left(\tau-\operatorname{int}\left[U \cap\left(f^{-1}(V)\right)^{*}\right]\right) \subseteq \tau-\operatorname{cl}\left(\tau-\operatorname{int}\left[U \cap f^{-1}(V)\right]^{*}\right)[4$, Theorem (2.3)]. Hence $f \mid U$ is quasi $I$-continuous.

Theorem 6. A function $f:(X, \tau, I) \rightarrow(Y, \sigma)$ is quasi $I$-continuous if its restrictions by an open cover $\left\{U_{i}: i \in \nabla\right\}$ of $X$ are quasi $I$-continuous.

Proof. Follows directly by using Propositions 1 and 7 .

Theorem 7. Any function is quasi I-continuous iff its graph is quasi I-continuous.

Proof. Let $f:(X, \tau, I) \rightarrow(Y, \sigma)$ be quasi $I$-continuous and $x \in X$ and $H$ is an open set in $X \times Y$ containing the graph image of $x, g(x)$. Then there exist $U \in \tau$ and $V \in \sigma$ such that $g(x)=(x, f(x)) \in U \times V \subseteq H$. By hypothesis of $f$, there exists $W \in Q I O(X, x)$ such that $f(W) \subseteq V$. Proposition 2 shows that $x \in U \cap W \in Q I O(X, \tau)$ which contained in $U$. So, $(U \cap W) \times V \subseteq U \times V \subseteq H$ and hence $g(U \cap W) \subseteq H$. This means that $g:(X, \tau, I) \rightarrow\left(X \times Y, \tau_{X \times y}\right)$ is quasi $I$-continuous. Conversely, let $x \in X$ and $V \in \sigma(f(x))$, then $X \times V \in \tau_{X \times y}$. Quasi $I$-continuity of $g$ shows that, there exists $W \in Q I O(X, x)$ such that $g(W) \subseteq X \times V$ the projection of both sides gives $f(W) \subseteq V$. Hence $f$ is quasi $I$-continuous and this completes the proof.

Recall that, $(X, \tau)$ is a quasi $I$-compact space if for every quasi: $I$-open cover $\left\{W_{i}\right.$ : $i \in \nabla\}$, there exists a finite $\nabla_{0}$ of $\nabla$ such that $X \cup\left\{W_{i}: i \in \nabla_{0}\right\} \in I$. 
Lemma 4. [11] For any function $f:(X, \tau, I) \rightarrow(Y, \sigma), f(I)$ is an ideal on $Y$.

Theorem 8. The image of quasi I-compact under a quasi I-continuous sjrjective function is $f(I)$-compact.

Proof. Let $f:(X, \tau, I) \rightarrow(Y, \sigma)$ be quasi $I$-continuous and $\left\{V_{i}: i \in \nabla\right\}$ be an open over of $Y$. Then $\left\{f^{-1}\left(V_{i}\right), i \in \nabla\right\}$ is a quasi $I$-open cover of $X$. From assumption, there exists a finite $\nabla_{0}$ of $\nabla$ such that $\left.X-\cup\right\}\left\{f^{-1}\left(V_{i}\right): i \in \nabla_{0}\right\} \in I$. Therefore, $Y-\cup\left\{V_{i}: i \in \nabla_{0}\right\} \in f(I)$ which shows that $(Y, \sigma)$ is $f(I)$-compact.

\section{References}

[1] M. E. Abd El-Monsef, S. N. El-Deeb and R. A. Mahmoud, $\beta$-open sets and $\beta$-continuous mappings, Bull. Fac. Sci., Assiut Univ., 12(1983), 77-90.

[2] M. E. Abd El-Monsef, E. F. Lashien and A. A. Nasef, On I-open sets and I-continuous functions, Kyungpook Math. J., 32(1992), 21-30.

[3] E. Hayashi, Topologies defined by local properties, Math. Ann. 156(1964), 205-215.

[4] D. Jankovic and T. R. Hamlett, New topologies from old via ideals, Amer. Math. Monthly, 97(1990), 295-310.

[5] D, Jankovic and T. R. Hamlett, Compatible extensions of ideals, Boll. U.M.I., 7(6-B)(1992), 453-465.

[6] K. Kuratowski, Topologies I, Warszawa, 1933.

[7] K. Kuratowski, Topologies Vol.I, Academic Press, New-York, 1966.

[8] N. Levine, Semi-open sets and semi-continuity in topological spaces, Amer. Math. Monthly, 70(1963), 36-41.

[9] R. A. Mahmoud and M. E. Abd El-Monsef, $\beta$-irresolute and $\beta$ topological invariant, J. Pakistan Academy of Sci, (1989), 285-296.

[10] A. S. Mashhour, M. E. El-Monsef and S. N. El-Deeb, On precontinuous and weak precontinuous mappings, Proc. Math. Phys. Soc. Egypt 53(1982), 47-53.

[11] R. L. Newcomb, Topologies which are compact modulo an ideal, Ph. D. dissertation, Univ. of Col. at Santa Barbara, 1967.

[12] O. njastad, On some classes of nearly open sets, Pacific J. Math., 15(1965), 961-970.

[13] O. Njastad, Remarks on topologies defined by local properties, Avh. Norske Vid. Akad. Oslo I(N.S), 8(1966), 1-16.

[14] D. V. Rancin, Compactness modulo an ideal, Soviet Math. Dokl., 13(1972), 193-197.

[15] R. Vaidyanathaswamy, Thelocalization theory in set topology, Proc. Indian Acad. Sci, 20(1945), 51-61.

[16] R. Vaidyanathaswamy, Set Topology, Chelsea, 2nd Edition, New-York, 1960.

${ }^{*}$ Department of Mathematics, Faculty of Science, Tanta University.

** Department of Mathematics, Faculty of Science, Menoufia University, Shebin El-Koom, Egypt.

*** Department of Mathematics, Faculty of Education, Suez-Canal University. 